syndromes and genetics: a review. J Med Genet. 2008;45(1):1-14.

3. Matsuda NM, Miller SM, Evora PR. The chronic gastrointestinal manifestations of Chagas disease. Clinics (Sao Paulo). 2009;64(12):1219-1224.

4. Moore SW, Johnson G. Acetylcholinesterase in Hirschsprung's disease. Pediatr Surg Int. 2005; 21(4):255-263

5. Wood JD. Enteric nervous system: reflexes, pattern generators and motility. Curr Opin Gastroenterol. 2008;24(2):149-158.

6. Linden DR, et al. Indiscriminate loss of myenteric neurones in the TNBS-inflamed guinea-pig distal colon. Neurogastroenterol Motil. 2005;17(5):751-760.

7. Mu Y, Lee SW, Gage FH. Signaling in adult neurogenesis. Curr Opin Neurobiol. 2010;20(4):416-423.

8. Kruger GM, Mosher JT, Bixby S, Joseph N, Iwashita
T, Morrison SJ. Neural crest stem cells persist in the adult gut but undergo changes in self-renewal, neuronal subtype potential, and factor responsiveness. Neuron. 2002;35(4):657-669.

9. Pham TD, Gershon MD, Rothman TP. Time of origin of neurons in the murine enteric nervous system. J Comp Neurol. 1991;314(4):789-798.

10. Chalazonitis A, et al. Bone morphogenetic protein regulation of enteric neuronal phenotypic diversity: relationship to timing of cell cycle exit. J Comp Neurol. 2008;509(5):474-492.

11. Joseph NM, He S, Quintana E, Kim Y-G, Núñez G, Morrison SJ. Enteric glia are multipotent in culture but primarily form glia in the adult rodent gut. J Clin Invest. 2011;121(9):3398-3411.

12. Laranjeira C, et al. Glial cells in the mouse enteric nervous system can undergo neurogenesis in response to injury. J Clin Invest. 2011;121(9):3412-3424.

13. Garcia AD, Doan NB, Imura T, Bush TG, Sofroniew MV. GFAP-expressing progenitors are the principal source of constitutive neurogenesis in adult mouse forebrain. Nat Neurosci. 2004;7(11):1233-1241.

14. Liu MT, Kuan YH, Wang J, Hen R, Gershon MD. 5-HT4 receptor-mediated neuroprotection and neurogenesis in the enteric nervous system of adult mice. J Neurosci. 2009;29(31):9683-9699.

15. Matsuyoshi H, et al. A 5-HT(4)-receptor activationinduced neural plasticity enhances in vivo reconstructs of enteric nerve circuit insult. Neurogastroenterol Motil. 2010;22(7):806-813.

16. Li Z, et al. Essential roles of enteric neuronal serotonin in gastrointestinal motility and the development/survival of enteric dopaminergic neurons. J Neurosci. 2011;31(24):8998-9009.

\title{
Parkin reinvents itself to regulate fatty acid metabolism by tagging CD36
}

\author{
Nada A. Abumrad ${ }^{1}$ and Darren J. Moore ${ }^{2}$ \\ ${ }^{1}$ Washington University School of Medicine, Department of Medicine, Center for Human Nutrition, St. Louis, Missouri, USA. \\ 2Ecole Polytechnique Fédérale de Lausanne (EPFL), Brain Mind Institute, School of Life Sciences, Lausanne, Switzerland.
}

\begin{abstract}
Parkinson disease (PD) is a relatively common neurodegenerative disorder characterized by the progressive degeneration of dopaminergic neurons in the substantia nigra. About $5 \%-10 \%$ of PD cases are inherited. Mutations in the Parkin gene, which encodes a protein that can function as an E3 ubiquitin ligase, are a common cause of familial PD. Such mutations act in a lossof-function manner and impair the ability of the encoded protein to mediate substrate ubiquitination, although the subsequent molecular pathway that precipitates neuronal degeneration is poorly defined. In this issue of the JCI, Kim and colleagues describe painstaking evidence using a number of dissecting approaches in intact animals and cultured cells to functionally link Parkin and the class B scavenger receptor CD36, suggesting a novel and complex connection between PD and fatty acid metabolism.
\end{abstract}

Parkinson disease (PD) affects $1 \%-2 \%$ of the world's population over the age of 65 years. It is more common in developed countries where people live longer. PD is characterized by the progressive degeneration of dopaminergic neurons in the substantia nigra, which leads to diminished striatal dopamine levels and the appearance of intracytoplasmic proteinaceous inclusions, termed Lewy bodies, in surviving brainstem neurons. The cardinal motor symptoms of PD include bradykinesia, resting tremor, muscular rigidity, and postural instability. There are also nonmotor symptoms, which include autonomic, cognitive, and psychiatric disturbances. About

Conflict of interest: The authors have declared that no conflict of interest exists.

Citation for this article: J Clin Invest. 2011; 121(9):3389-3392. doi:10.1172/JCI59219.
$5 \%-10 \%$ of PD cases are inherited, with at least 16 chromosomal loci (PARK1-16) associated with these familial forms of disease (1). Mutations in the $\alpha$-synuclein gene (SNCA, which falls into both the PARK1 and PARK4 loci) and the leucine-rich repeat kinase 2 gene (LRRK2, which accounts for the PARK8 locus) unambiguously cause autosomal dominant PD, whereas mutations in the Parkin gene (which accounts for the PARK2 locus), the PTEN-induced putative kinase 1 gene (PINK1, which accounts for the PARK6 locus), the DJ-1 gene (which accounts for the PARK7 locus), and the ATPase type 13A2 gene (ATP13A2, which accounts for the PARK9 locus) cause autosomal recessive forms of the disease (1). Idiopathic PD is considered to result from a combination of genetic susceptibility, aging, and environmental factors (for example, pesticides) (2).

\section{Parkin: a ubiquitin ligase linked to PD}

Mutations in the Parkin gene on chromosome 6 cause autosomal recessive, juvenile-onset parkinsonism (AR-JP), the most common form of early-onset disease $(3,4)$. The Parkin gene encodes a 465 amino acid protein consisting of a ubiquitin-like domain and a RING-box domain containing two RING finger motifs separated by an in-between-RING finger motif (Figure 1 and ref. 5). Similar to other RING-box proteins, Parkin can function as an E3 ubiquitin ligase to catalyze, in cooperation with E1-activating and E2-conjugating enzymes, the covalent attachment of the carboxyl terminal glycine residue of ubiquitin to an internal lysine residue of protein substrates (5). Parkin can link substrates to chains of ubiquitin polymerized through different lysine residues of the ubiquitin components, with K48-linked chains targeting substrates for proteasomal degradation and K63-linked chains acting as a nondegradative signal (5). Parkin can also mediate monoubiquitination of proteins and itself (6). Familial mutations in Parkin act in a loss-of-function manner and impair substrate ubiquitination, although the subsequent molecular pathway that precipitates neuronal degeneration is poorly defined. In this issue of the JCI, Kim et al. have identified a new substrate of Parkin-mediated ubiquitination that provides a novel and 


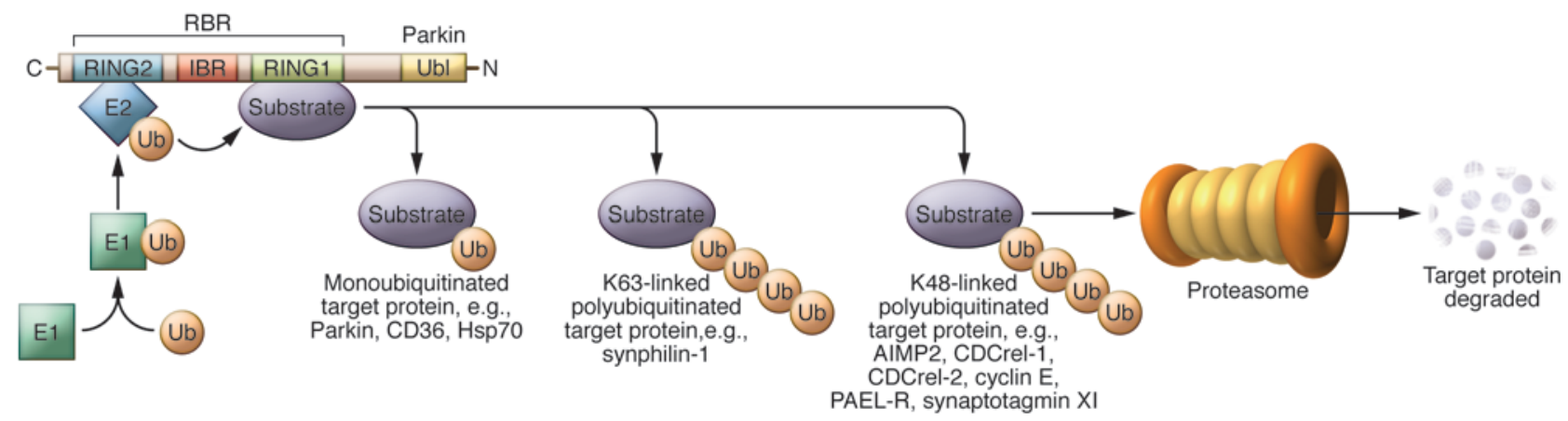

\section{Figure 1}

Substrates of Parkin-dependent ubiquitination. Parkin contains a ubiquitin-like (Ubl) domain and a RING-box (RBR) domain consisting of two RING finger motifs separated by an in-between-RING finger domain (IBR). Parkin cooperates with E1-activating and E2-conjugating enzymes to covalently modify protein substrates with ubiquitin, either via alternatively linked polyubiquitin chains or via monoubiquitination. Polyubiquitination via K48-linked chains serves to target substrates for proteasomal degradation, whereas K63-linked polyubiquitination and monoubiquitination serve as nondegradative signals. A broad range of substrates is ubiquitinated by Parkin, often being targeted for degradation (i.e., PAEL-R, AIMP2/p38, CDCrel-1, and cyclin E), whereas others are degradation independent (i.e., Hsp70 and synphilin-1). The lipid transporter CD36 is the latest substrate identified for Parkin and is stabilized by monoubiquitination (7), providing a novel mechanism for the regulation of FA uptake.

complex connection between fatty acid (FA) metabolism and PD (7).

\section{Parkin finds a new substrate}

A number of substrates have been identified for Parkin (Figure 1), including numerous proteins of the outer mitochondrial membrane $(5,8)$. However, impaired ubiquitination of these has yet to be linked molecularly to PD. The study by Kim et al. (7) identifies the class B scavenger receptor CD36 (also known as FA translocase [FAT]) as a new substrate of Parkin-mediated ubiquitination and proposes a potentially broad function of Parkin in the regulation of FA metabolism. CD36 is a 472 -amino acid transmembrane protein that binds with high affinity to a number of lipid ligands including long chain FAs, anionic phospholipids, and native or modified lipoproteins. CD36 also recognizes a number of nonlipid ligands such as thrombospondin- 1 , collagen, and amyloid- $\beta$. Interaction of CD36 with its ligands elicits a range of intracellular signaling processes involving Src and MAP kinases that integrate lipid metabolism and inflammation and also involve CD36 in pathways related to oxidative stress, angiogenesis, platelet hyperactivity, phagocytosis, and cell migration $(9,10)$. Studies in CD36-deficient mice (11) and humans (12) have linked CD36 to FA and glucose utilization. Common polymorphisms in the CD36 gene contribute to individual variability in blood lipids (13) and to differences in platelet function (14). They may also influence susceptibility to the metabolic syndrome (13) and diabe- tes (15). Overall, although CD36 is clearly multifunctional, data currently available in humans mainly implicate the protein in FA and lipoprotein metabolism and associated abnormalities.

The regulation of CD36 by Parkin uncovered by Kim et al. (7) is consistent with epidemiologic studies linking altered lipid metabolism to $\operatorname{PD}(16,17)$ and with evidence that abnormalities in lipid processing contribute to degenerative brain diseases (18). Indeed, these earlier findings are what led Kim and colleagues to investigate the possibility that Parkin regulates systemic lipid metabolism by characterizing the global metabolic responses of WT and Parkin-deficient (Parkin ${ }^{-/}$) mice to high-fat diet (HFD) (7). As expected, the WT mice gained weight on the diet and developed insulin resistance and lipid accumulation in the liver, which was associated with parallel increases in lipid transport proteins and Parkin. In contrast, the Parkin ${ }^{-/-}$mice were resistant to all these metabolic changes. CD36 was among the lipid transport proteins altered by HFD in the WT but not Parkin $^{-/-}$mice, and CD36 levels paralleled those of Parkin in multiple tissues. Importantly, rescue of CD36 expression in the liver of Parkin ${ }^{-/}$mice increased hepatic fat uptake while rescue of Parkin expression restored endogenous CD36 levels and lipid accumulation. Gain- and loss-of-function studies in cultured cells documented that Parkin regulates CD36 levels and FA uptake, while immunoprecipitation experiments suggested a physical interaction between CD36 and Parkin. Reduction of FA uptake was also observed in B cells derived from patients harboring heterozygous Parkin mutations that associate with earlyonset parkinsonism.

Kim et al. (7) went on to show that the regulation of CD36 by Parkin is mediated via its monoubiquitination, which they propose stabilizes CD36 at the plasma membrane. This contrasts with FAs, which were previously shown to induce polyubiquitination of CD36 and target it for proteasomal degradation. The lysine residues within CD36 that are ubiquitinated upon exposure to FAs are K469 and K472 in the carboxyl terminus of the protein (10). One of these residues is likely targeted by Parkin, since these are the only lysines present in the cytoplasmic domains of CD36. These residues could constitute a site where the influences of FAs and Parkin on CD36 are integrated. Consistent with FAs and Parkin competing to regulate CD36 levels, Kim et al. (7) found that Parkin prevents FAmediated reduction in CD36 levels. FA induction of CD36 polyubiquitination and degradation acts to limit cellular toxicity from high FAs and would be blunted in the presence of Parkin. Whether this means that Parkin contributes to abnormalities of FA utilization under conditions of high FAs remains to be determined. However, it is clear from the data generated by Kim et al. (7) that Parkin is a player in the regulation of lipid metabolism, especially considering that its expression is not limited to the brain and is observed in a number of metabolically active tissues including the heart and skeletal muscle, where it is particularly 


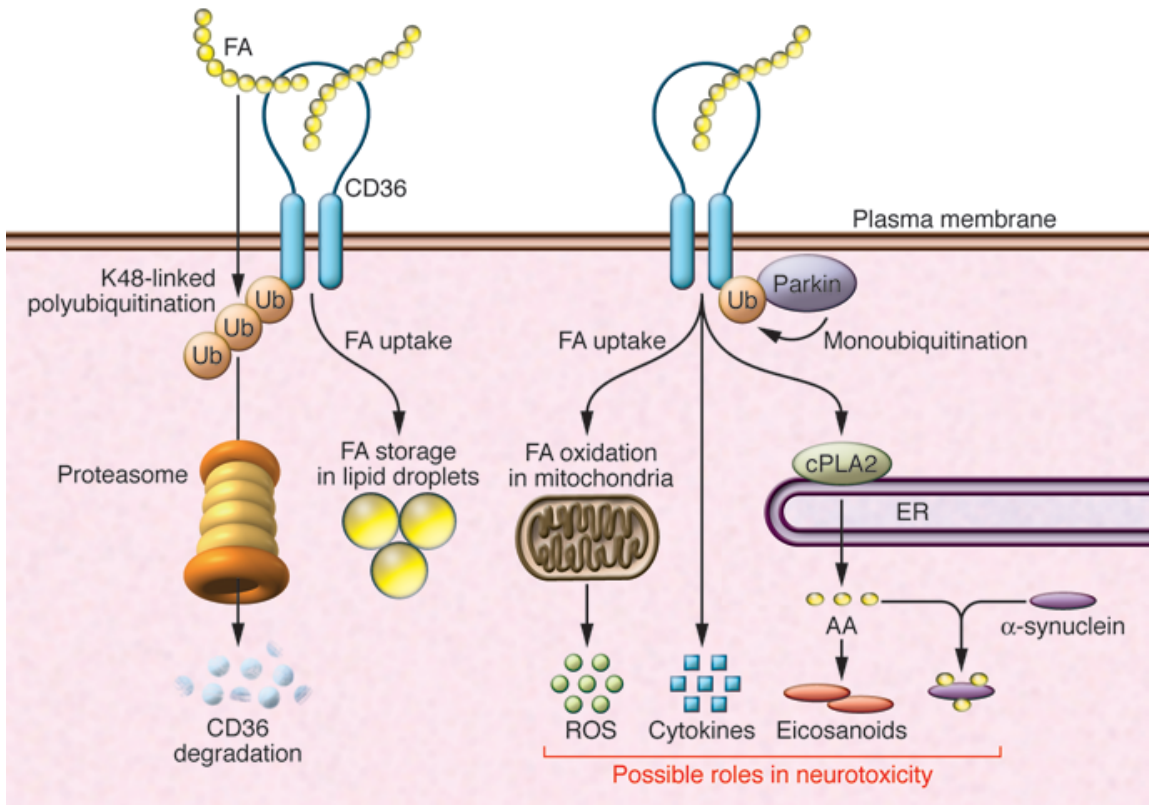

\section{Figure 2}

Potential functional interactions among Parkin, CD36, and the PD-associated protein $\alpha$-synuclein. Parkin monoubiquitinates CD36, stabilizing it and increasing its level at the plasma membrane. This is in contrast to long-chain FAs, which induce CD36 polyubiquitination and target CD36 for proteosomal degradation. At the plasma membrane, CD36 facilitates uptake of FAs, which are stored into lipid droplets or oxidized by mitochondria. Under conditions of cellular stress, CD36 is involved in $\mathrm{Ca}^{2+-}$ and ERK1/2-mediated activation of cPLA2 to release arachidonic acid (AA) from endoplasmic reticulum and nuclear membrane phospholipids. Brain $A A$ is involved in the regulation of synaptic transmission and is also converted by microglia to bioactive eicosanoids, which can have pleiotropic effects on inflammation. Excess production of AA-derived metabolites, ROS (from mitochondria), and cytokines (CD36 signaling contributes to cytokine production) by the activated microglia can lead to neurotoxicity. Binding of AA by $\alpha$-synuclein could modulate its effects and its metabolic conversion to eicosanoids.

abundant. This interpretation would be consistent with previous reports of a role for Parkin in oxidative metabolism and in maintaining mitochondrial quality control. Identified first in Drosophila, where it was reported that Parkin-null flies cannot climb or fly and exhibit reduced body mass (19), it was later shown in various mammalian cell types that under stressful conditions, Parkin selectively translocates to damaged mitochondria to ubiquitinate mitochondrial proteins and induce mitophagy (8). It is worth noting that the Parkin substrate CD36 has been identified in isolated mitochondrial preparations, especially under states of high-energy demand such as exercise, where it would facilitate FA access to metabolizing enzymes (20).

\section{FAs and PD}

The paper by Kim et al. (7) shows that Parkin is lipid sensitive and is regulated by dietary fat intake. So, does FA metabolism modulate susceptibility to PD? Emerging epidemiological evidence lends some support to this. In particular, increased dietary intake of omega-3 polyunsaturated FAs (n-3 PUFAs) has been associated with a decreased risk of PD (16), while increased PD risk has been associated with a high intake of the n-6 PUFA arachidonic acid (21). Dietary n-3 PUFAs also provide neuroprotection in toxin-induced rodent models of parkinsonism (16). These studies suggest a role for FA metabolism in the maintenance and survival of nigrostriatal pathway dopaminergic neurons. They also touch on the importance of arachidonic acid metabolism in the brain and how its dysregulation may associate with disease (22). Arachidonic acid, released from membrane phospholipid by phospholipases, notably cytosolic phospholipase A2 (cPLA2), and its metabolites are involved in the regulation of synaptic transmission, and this may be modulated by the PD-associated protein $\alpha$-synuclein, which sequesters the released arachidonic acid (23). Coincidentally, the novel Parkin substrate CD36, iden- tified by Kim et al. (7), is also involved in the regulation of arachidonic acid levels. CD36 facilitates arachidonic acid release by virtue of its role in activating membrane calcium channels and cPLA2 (24). It is tempting therefore to speculate that Parkin, CD36, and $\alpha$-synuclein (Figure 2) may interact to control arachidonic acid metabolism in the brain. It is known that excess arachidonic acid release by activated brain microglia results in the formation of neurotoxic compounds such as prostaglandins, cytokines, and reactive oxygen species (18). Misfolded or aggregated $\alpha$-synuclein (like amyloid- $\beta$ ) would potentiate these events. Studies using transgenic mice or cultured primary microglia overexpressing human $\alpha$-synuclein mutations showed early microglial activation that was partially inhibited in microglia derived from CD36-deficient mice (25).

In summary, the paper by Kim et al. (7) brings novel insight into the well-known connection between neurodegenerative disorders and lipid metabolism. By documenting the clear link between Parkin and CD36, it introduces a new area of research with abundant possibilities. In addition, the paper shows that Parkin levels are regulated by lipid, an important finding that provides a concrete avenue by which dietary fat intake can have an impact on PD etiology. Possibly, we should ask, does aging increase PD susceptibility via the associated alterations in lipid metabolism?

\section{Acknowledgments}

Work in the lab of N.A. Abumrad related to CD36 ubiquitination by FAs and its role in arachidonic acid release is supported by NIHI grants P01 HL057278, DK33301, and DK56351.

Address correspondence to: Nada A. Abumrad, Department of Medicine, Campus Box 8031, Washington University School of Medicine, St. Louis, Missouri 63110, USA. Phone: 314.747.0348; Fax: 314.444.3432; E-mail: nabumrad@wustl.edu.

1. Gasser T. Mendelian forms of Parkinson's disease. Biochim Biophys Acta. 2009;1792(7):587-596

2. Wirdefeldt K, Adami HO, Cole P, Trichopoulos D, Mandel J. Epidemiology and etiology of Parkinson's disease: a review of the evidence. Eur J Epidemiol. 2011;26(suppl 1):S1-S58.

3. Kitada T, et al. Mutations in the Parkin gene cause autosomal recessive juvenile parkinsonism. Nature. 1998;392(6676):605-608.

4. West AB, Maidment NT. Genetics of Parkin-linked disease. Hum Genet. 2004;114(4):327-336.

5. Moore DJ. Parkin: a multifaceted ubiquitin ligase. Biochem Soc Trans. 2006;34(pt 5):749-753.

6. Moore DJ, West AB, Dikeman DA, Dawson VL, Dawson TM. Parkin mediates the degradation- 
independent ubiquitination of Hsp70. J Neurochem 2008;105(5):1806-1819.

7. Kim K-Y, et al. Parkin is a lipid-responsive regulator of fat uptake in mice and mutant human cells. J Clin Invest. 2011;121(9):3701-3712.

8. Chan NC, et al. Broad activation of the ubiquitin proteasome system by Parkin is critical for mitoph agy. Hum Mol Genet. 2011;20(9):1726-1737.

9. Silverstein RL, Febbraio M. CD36, a scavenger receptor involved in immunity, metabolism, angiogenesis, and behavior. Sci Signal. 2009;2(72):re3.

10. Su X, Abumrad NA. Cellular fatty acid uptake: a pathway under construction. Trends Endocrinol Metab. 2009;20(2):72-77.

11. Hajri T, Han XX, Bonen A, Abumrad NA. Defective fatty acid uptake modulates insulin responsiveness and metabolic responses to diet in CD36-null mice. J Clin Invest. 2002;109(10):1381-1389.

12. Yamashita S, et al. Physiological and pathological roles of a multi-ligand receptor CD36 in atherogenesis; insights from CD36-deficient patients. $\mathrm{Mol}$ Cell Biochem. 2007;299(1-2):19-22.

13. Love-Gregory L, et al. Common CD36 SNPs reduce protein expression and may contribute to a pro- tective atherogenic profile. Hum Mol Genet. 2011; 20(1):193-201.

14. Ghosh A, et al. Platelet CD36 surface expression levels affect functional responses to oxidized LDL and are associated with inheritance of specific genetic polymorphisms. Blood. 2011;117(23):6355-6366.

15. Lepretre F, et al. A CD36 nonsense mutation associated with insulin resistance and familial type 2 diabetes. Hum Mutat. 2004;24(1):104.

16. Bousquet M, Calon F, Cicchetti F. Impact of omega3 fatty acids in Parkinson's disease [published online ahead of print March 23, 2011]. Ageing Res Rev. doi:10.1016/j.arr.2011.03.001.

17. de Lau LM, Bornebroek M, Witteman JC, Hofman A, Koudstaal PJ, Breteler MM. Dietary fatty acids and the risk of Parkinson disease: the Rotterdam study. Neurology. 2005;64(12):2040-2045.

18. Farooqui T, Farooqui AA. Lipid-mediated oxidative stress and inflammation in the pathogenesis of Parkinson's disease. Parkinsons Dis. 2011;2011:247467.

19. Greene JC, Whitworth AJ, Kuo I, Andrews LA, Feany MB, Pallanck LJ. Mitochondrial pathology and apoptotic muscle degeneration in Drosophila Parkin mutants. Proc Natl Acad Sci U S A. 2003;
100(7):4078-4083.

20. Smith BK, et al. FAT/CD36 is located on the outer mitochondrial membrane, upstream of long-chain acyl-CoA synthetase, and regulates palmitate oxidation. Biochem J. 2011;437(1):125-134

21. Miyake Y, et al. Dietary fat intake and risk of Parkinson's disease: a case-control study in Japan. J Neurol Sci. 2010;288(1-2):117-122.

22. Farooqui AA, Horrocks LA, Farooqui T. Interactions between neural membrane glycerophospholipid and sphingolipid mediators: a recipe for neural cell survival or suicide. J Neurosci Res. 2007; 85(9):1834-1850.

23. Darios F, Ruiperez V, Lopez I, Villanueva J, Gutierrez LM, Davletov B. Alpha-synuclein sequesters arachidonic acid to modulate SNARE-mediated exocytosis. EMBO Rep. 2011;11(7):528-533.

24. Kuda O, et al. CD36 Protein Is Involved in Storeoperated Calcium Flux, Phospholipase A2 Activation, and Production of Prostaglandin E2. J Biol Chem. 2011;286(20):17785-17795.

25. Su X, Federoff HJ, Maguire-Zeiss KA. Mutant alphasynuclein overexpression mediates early proinflammatory activity. Neurotox Res. 2009;16(3):238-254.

\title{
Peripheral glucose homeostasis: does brain insulin matter?
}

\author{
Barry E. Levin ${ }^{1,2}$ and Robert S. Sherwin ${ }^{3}$
}

\begin{abstract}
${ }^{1}$ Neurology Service, Veterans Administration Medical Center, East Orange, New Jersey, USA. 2Department of Neurology and Neurosciences,
New Jersey Medical School, University of Medicine and Dentistry of New Jersey, Newark, New Jersey, USA. ${ }^{3}$ Department of Medicine,

Section of Endocrinology and Metabolism, Yale Center for Clinical Investigation, Yale University School of Medicine, New Haven, Connecticut, USA.
\end{abstract}

\begin{abstract}
Much controversy surrounds the relative role of insulin signaling in the brain in the control of hepatic glucose metabolism. In this issue of the JCI, Ramnanan and colleagues demonstrate that arterial infusion of insulin into the brains of dogs reduces net hepatic glucose output without altering endogenous glucose production. However, this effect was modest and required both prolonged fasting and prolonged exposure of the brain to insulin, raising doubts about the overall physiological relevance of insulin action in the brain on hepatic glucose metabolism. Given the dominant direct role that insulin plays in inhibiting glucose production in the liver, we suggest that the main effect of central insulin on hepatic glucose metabolism may be more chronic and assume greater significance either when portal insulin is deficient, as occurs during exogenous insulin treatment of type 1 diabetes, or when chronic hyperinsulinemia and central insulin resistance develops, as occurs in type 2 diabetes.
\end{abstract}

Insulin controls nutrient and metabolic homeostasis via effects on the liver, muscle, and adipose tissue. After a meal, insulin acts on the liver to inhibit net hepatic glucose output (NHGO) - the balance between hepatic glucose production (HGP), which occurs via gluconeogenesis and glycoge-

Conflict of interest: Robert S. Sherwin has stock options from Insulet and receives honoraria from Novartis and MannKind for service on data safety monitoring boards.

Citation for this article: J Clin Invest. 2011; 121(9):3392-3395. doi:10.1172/JCI59653. nolysis, and hepatic glucose uptake, which includes glycogen synthesis. In humans (1) and in large mammals such as the dog (2), the direct action of insulin on the liver plays a dominant role in suppressing NHGO. However, studies in rodents suggest that insulin can also act within the brain to alter hepatic glucose metabolism, primarily by suppressing HGP (Figure 1 and refs. refs. 3 , 4). In this issue of the JCI, Ramnanan and colleagues show that raising brain insulin levels in dogs using the physiologically relevant route of carotid and verte- bral arterial infusions inhibits NHGO by increasing hepatic uptake of glucose and its incorporation into glycogen (Figure 1 and ref. 5). HGP was not substantially altered, although the expression of gluconeogenic genes in liver was diminished. However, unlike the transient (approximately 1-2 hours) rise in insulin and fall in glucagon levels in the portal circulation that follows most carbohydrate-rich meals, the insulin infusions performed by Ramnanan and colleagues were carried out for 3-4 hours and the dogs were fasted for 42 hours; even under these conditions, only a relatively modest inhibition of NHGO was observed. While the work of Ramnanan and colleagues (5) confirms that insulin can act on the brain to alter hepatic glucose metabolism in dogs, it does not settle the question about the relative importance of central insulin signaling in mediating NHGO under physiological conditions in humans and other mammals.

\section{Insulin and the brain}

The brain is an insulin-sensitive organ; various studies have shown that insulin action in the brain affects energy and glu- 